\title{
Colección de Mastozoología del Museo de La Salle - Bogotá (MLS)
}

\author{
Cristian Alexander Cruz-Rodríguez ${ }^{1 *(\mathbb{D})}$, Julieth Stella Cárdenas²(D) \\ 1 Instituto de Investigación de Recursos Biológicos Alexander von Humboldt. Avenida Circunvalar \# 16 - 20, Bogotá D.C. \\ Colombia. \\ 2 Museo de La Salle, Universidad de La Salle. Carrera 2 \# 10-70, Bogotá D.C. Colombia. \\ * Correspondencia: cruzrodriguezcristian@gmail.com
}

\section{Resumen}

La colección de mastozoología del Museo de La Salle - Bogotá (MLS) alberga 2540 especímenes de mamíferos pertenecientes a 17 órdenes, 71 familias, 236 géneros y 389 especies. Los especímenes proceden de 26 departamentos del país y algunos del extranjero. La colección es la más antigua de este tipo en Colombia y contiene el mamífero colombiano más antiguo depositado en colecciones de nuestro país, colectado en marzo de 1903 en Rionegro, Antioquia.

Palabras clave: Bogotá, especímenes, Hermanos Lasallistas, holotipo, sinónimos.

\section{Abstract}

The mastozoological collection of the Museo de La Salle - Bogotá (MLS) holds 2540 mammal specimens belonging to 17 orders, 71 families, 236 genera and 389 species. The specimens come from 26 departments in the country and some from abroad. The collection is the oldest of its kind in Colombia and contains the oldest Colombian mammal deposited in collections in our country, collected in March 1903 in Rionegro, Antioquia.

Key words: Bogotá, Lasallian Brothers, holotype, specimens, synonyms.

La colección de mastozoología del Museo de La Salle - Bogotá (MLS) fue fundada en 1904 y se ubica en La Candelaria, Centro Histórico de la Ciudad de Bogotá D.C. (Figura 1). La colección, es el fiel reflejo de las exploraciones y los grandes descubrimientos que se llevaron a cabo a lo largo del siglo XX por los Hermanos Lasallistas en Colombia, investigadores innatos que establecieron las primeras colecciones en el país; fue tan valiosa su participación en la mastozoología colombiana, que diferentes especies fueron dedicadas a la obra de los directores del Museo como lo son Glossophaga apolinari Allen 1916 (sinónimo reciente de Anoura peruana); Thrinacodus apolinari Allen 1914 (sinónimo reciente de Olallamys albicaudus); Trinycteris nicefori Sanborn 1949; Sylvilagus apollinaris Thomas 1920 y otras fueron descritas por ellos, como el oso de anteojos de La Salle Tremarctos lasallei Nicéforo María 1924 (sinónimo reciente de Tremarctos ornatus).

También se destaca el ejemplar MLS 242 (Lontra longicaudis Olfers 1818), el cual es el 
mamífero colombiano más antiguo depositado en colecciones de nuestro país (colectado en marzo de 1903 en Rionegro, Antioquia).

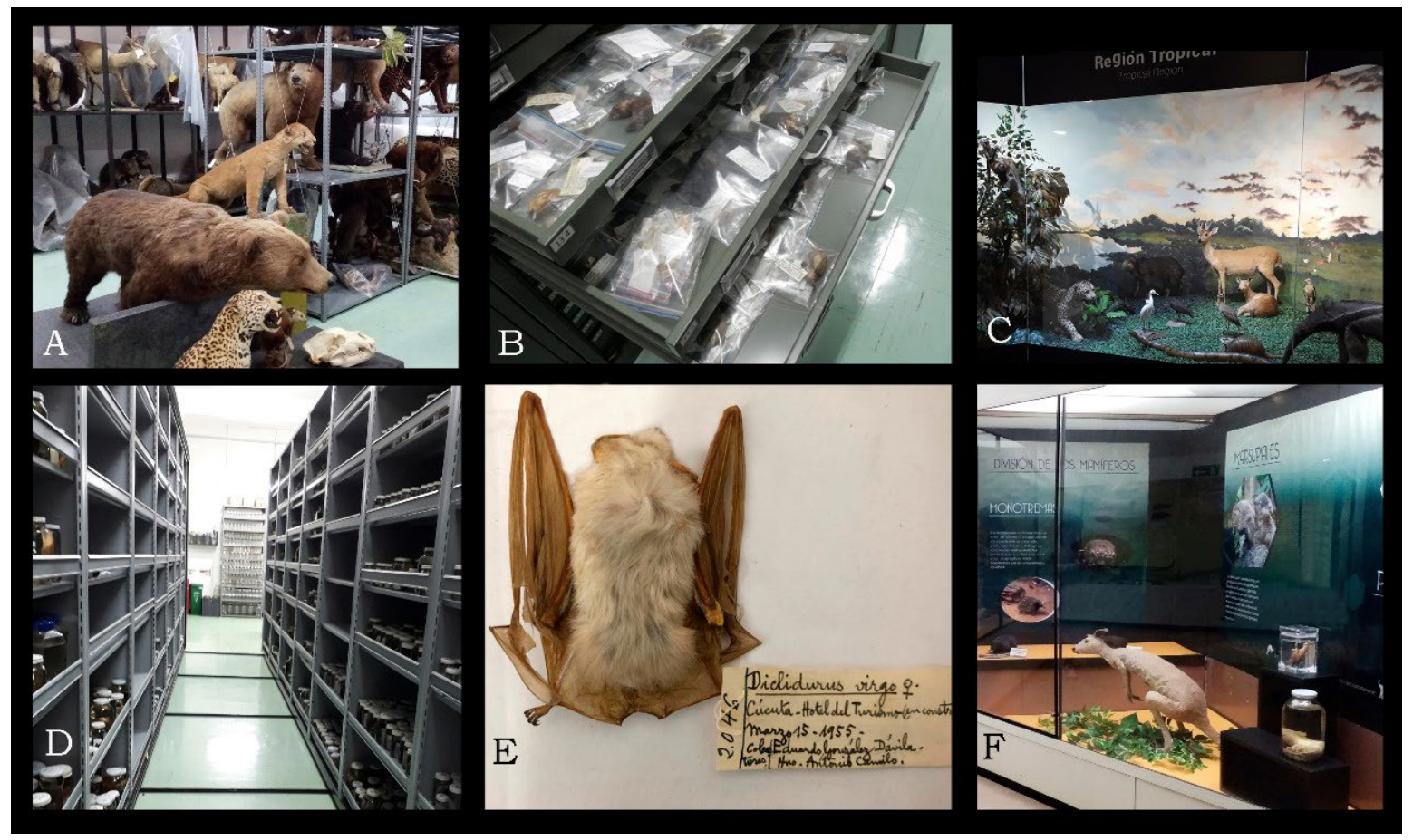

FIGURA 1. Componentes de la colección de mastozoología del Museo de La Salle - Bogotá (MLS). A) Depósito de grandes mamíferos y taxidermias. B) Depósito y unidades de almacenamiento de la colección en seco de quirópteros; C) Exposición permanente de la región tropical; D) Depósito de la colección en líquido; E) Ejemplar de Diclidurus albus en seco; F) Exposición permanente de la división de los mamíferos.

En la actualidad, la colección alberga 2540 especímenes de mamíferos pertenecientes a 17 órdenes, 71 familias, 236 géneros y 389 especies (Tabla 1). Todos los especímenes se encuentran catalogados y 8 están pendientes por ser ingresados a la colección. Los órdenes mejor representados son Chiroptera (1249 especímenes) y Rodentia (486) con 112 y 124 especies respectivamente (Tabla 1). Los especimenes proceden de Amazonas (34), Antioquia (141), Arauca (1), Archipiélago De San Andrés, Providencia y Santa Catalina (17), Atlántico (37), Bogotá, D.C. (28), Bolívar (27), Boyacá (94), Caldas (56), Caquetá (102), Casanare (24), Cauca (21), Cesar (8), Chocó (6), Córdoba (16), Cundinamarca (374), Huila (4), La Guajira (43), Magdalena (12), Meta (250), Norte de Santander (396), Risaralda (9), Santander (184), Sucre (3), Tolima (120), Valle del Cauca (16), Vaupés (4) y fuera de Colombia (513). Un total de 10 especímenes se encuentran sin identificar y tan solo 14 están aún a nivel de familia.

Especímenes de la colección (MLS) han sido empleados en al menos 15 publicaciones científicas (Nicéforo María 1924, 1947, 2004; Apolinar María 1913a,b; Apolinar María 1914a,b; Apolinar María 1915; Gonzalo Manuel 1956; Rodríguez-Posada \& Cárdenas-González 2012; Muñoz-Saba et al. 2013; Racero-Casarrubia et al. 2015; Morales-Martínez 2017; RamírezChaves et al. 2019; Torres-Martínez et al. 2019; Leuro-Robles et al. en prensa). 
Mastozoológica del Museo de La Salle (MLS).

\begin{tabular}{lcccc}
\hline \multicolumn{1}{c}{ Orden } & Familias & Géneros & Especies & Número de especímenes \\
\hline Monotremata & 2 & 2 & 2 & 2 \\
Diprodontia & 1 & 1 & 1 & 2 \\
Peramelemorphia & 1 & 1 & 1 & 1 \\
Didelphimorphia & 2 & 10 & 12 & 91 \\
Paucituberculata & 1 & 1 & 1 & 1 \\
Cingulata & 2 & 3 & 4 & 22 \\
Pilosa & 4 & 5 & 10 & 83 \\
Hyracoidea & 1 & 1 & 1 & 1 \\
Eulipotyphla & 3 & 7 & 10 & 20 \\
Chiroptera & 10 & 54 & 112 & 1249 \\
Carnivora & 10 & 33 & 50 & 266 \\
Perissodactyla & 2 & 2 & 2 & 6 \\
Artiodactyla & 6 & 18 & 22 & 97 \\
Cetacea & 2 & 2 & 2 & 6 \\
Primates & 8 & 17 & 31 & 177 \\
Rodentia & 14 & 77 & 124 & 486 \\
Lagomorpha & 2 & 2 & 4 & 20 \\
Sin identificar & - & - & - & 10 \\
Total & 71 & 236 & 389 & 2540 \\
\hline
\end{tabular}

\section{AGRADECIMIENTOS}

Agradecemos al director Fernando Sarmiento Parra, a Goretti Leuro-Robles, así como a todo el personal que trabaja en el Museo de La Salle por su apoyo en el proceso. Adicionalmente, agradecemos a Héctor Ramírez-Chaves por sus aportes en la presente nota, así como su liderazgo en la iniciativa.

\section{REFERENCIAS}

Apolinar María H. 1913a. Catálogo explicativo del Museo del Instituto de La Salle. Boletín de la Sociedad de Ciencias Naturales del Instituto de La Salle 1:20-25.

Apolinar María H. 1913b. Catálogo explicativo del Museo del Instituto de La Salle. Boletín de la Sociedad de Ciencias Naturales del Instituto de La Salle 1:52-58.

Apolinar María H. 1914a. Catálogo explicativo del Museo del Instituto de La Salle. Boletín de la Sociedad de Ciencias Naturales del Instituto de La Salle 2:58-61.

Apolinar María H. 1914b. Sección Zoológica. Boletín de la Sociedad de Ciencias Naturales del Instituto de La Salle 2:231-235.

Apolinar María H. 1915. Sección de Zoología, especies nuevas. Boletín de la Sociedad de Ciencias Naturales del Instituto de La Salle 3:114. 
Gonzalo Manuel H. 1956. Un Científico y el Museo de La Salle. Boletín del Instituto de La Salle 196: $52-56$.

Leuro-Robles N, Cruz-Rodríguez CA, Cárdenas-Hincapié JS. En prensa. Salvaguardando el patrimonio biológico de la sociedad. Evaluación del estado de salud de la colección de murciélagos (Chiroptera) del Museo de La Salle, Bogotá. Revista Academia Colombiana de Ciencias Exactas Físicas y Naturales.

Morales-Martínez D. 2017. Taxonomía y sistemática de los murciélagos del género, Micronycteris Gray, 1982 (Chiroptera: Phyllostomidae) en Colombia. Tesis de Maestría, Universidad Nacional de Colombia, Facultad de Ciencias, Instituto de Ciencias Naturales. Bogotá, Colombia.

Muñoz-Saba Y, Hoyos M, Casallas D, Calvo N. 2013. Murciélagos. Orden Chiroptera. En: Muñoz-Saba Y, González-Sánchez I, Calvo-Roa N, editores. Cavernas de Santander Colombia. Guía de Campo, Serie de Guías de Campo del Instituto de Ciencias No. 13. Universidad Nacional de Colombia, Bogotá. p. 241-248.

Nicéforo María H. 1924. Nueva especie de úrsidos colombianos. Boletín de la Sociedad Colombiana de Ciencias Naturales 13:113-117.

Nicéforo María H. 1947. Quirópteros de Colombia. Boletín del Instituto La Salle 34:34-47.

Nicéforo María H. 2004. Quirópteros Museo La Salle. Bogotá, Colombia. Grupo editorial Ediciones Unisalle, Bogotá, Colombia.

Racero-Casarrubia J, Ballesteros-Correa J, Pérez-Torres J. 2015. Mamíferos del departamento de Córdoba-Colombia: historia y estado de conservación. Biota Colombiana 16:128-148.

Ramírez-Chaves HE, Torres-Martínez MM, Noguera-Urbano EA, Passos FC, Colmenares-Pinzón JE. 2019. State of knowledge and potential distribution of the Colombian endemic Brown Hairy Dwarf Porcupine Coendou vestitus (Mammalia: Rodentia). Mammalian Biology 90:1-11. https://doi.org/10.1016/j.mambio.2019.09.012

Rodríguez-Posada M, Cárdenas-González C. 2012. El murciélago de visera Sphaeronycteris toxophyllum Peters, 1882 (Chiroptera: Phyllostomidae) en Colombia. Chiroptera Neotropical 18:1115-1122.

Torres-Martínez MM, Ramírez-Chaves HE, Noguera-Urbano EA, Colmenares-Pinzón JE, Passos FC, García J. 2019. On the distribution of the Brazilian porcupine Coendou prehensilis (Erethizontidae) in Colombia. Mammalia 83:290-297. https://doi.org/10.1515/mammalia2018-0043.

Editor: Héctor E. Ramírez-Chaves

Recibido: 2020-04-14

Revisado: 2020-04-21

Aceptado: 2020-04-21

Publicado: 2020-06-16 